\title{
Evaluation of Huffman Algorithm for Compression Standards
}

\author{
Swati D. Ghule ${ }^{1}$ and Anup Girdhar ${ }^{2}$ \\ ${ }^{1} M C A$ Department, P.E. S. Modern College of Engineering, Pune - 5, SPPU, India \\ ${ }^{2}$ Guide, Computer Science Department, Tilak Maharashtra Vidyapeeth, Pune, India
}

\section{ABSTRACT}

Data Compression is a technique to reduce the quantity of data without reducing the quality of the data. The transferring and storing of compressed data is much faster and more efficient than original uncompressed data. There are various techniques and standards of data compression are available. In this paper we implemented and tested Huffman algorithms. Our implementation results shows that compression ratio and the performance of the Huffman coding is higher for text and rich text format. In addition, implementation of Huffman coding is very easy.

KEY WORDS: HUFFMAN ALGORITHM, COMPRESSION RATIO, COMPRESSION TIME, LOSSLESS, LOSSY.

\section{INTRODUCTION}

In the field of computer science and information technology, Huffman coding is known as entropy encoding algorithm which is used for lossless data compression. The term entropy means the use of a variable-length code table for encoding a source symbol like character in file where the variable-length code table has been generated in a particular way based on the estimated frequency of occurrence for each possible value of the source symbol. Huffman coding is based on the frequency of occurrence of data symbols. The principle here is to use lower number of bits to encode the data that occurs more frequently. The average length of a Huffman code depends on the frequency with which the source produces each symbol from its alphabet.

Biosc Biotech Res Comm P-ISSN: 0974-6455 E-ISSN: 2321-4007

\section{crossef}

Identifiers and Pagination

Year: 2021 Vol: 14 No (5) Special Issue

Pages: $44-46$

This is an open access article under Creative

Commons License Attribn 4.0 Intl (CC-BY).

DOI: $h t t p: / / d x$.doi.org/10.21786/bbrc/14.5/9
In Huffman code dictionary, each data symbol is associated with code word, has the property that no codeword in the dictionary is a prefix of any other code word in the dictionary. The basis for this coding is a code tree according to Huffman, which assigns short code words to the symbols which are frequently used and long code words to the symbols which are rarely used. Note that the form in which Huffman tables are represented in the data stream is an indirect specification which can be used by the decoder to construct the tables themselves before the decompression. The Huffman algorithm for building the encoding follows this algorithm each symbol is a leaf and a root. The flowchart of the Huffman algorithm is depicted in figure.

We have studied, implemented, and tested Huffman coding on different file formats. Our experimental results show that performance of the Huffman coding is higher for text and rich text format. In addition, implementation complexity of Huffman coding is less. The remaining paper is organized as follows. Section 2 the need / Importance, Section 3 is Statement of Problem and 4 is Hypothesis. Section 5 is Research Methodology which discusses implementation of the algorithm and standard. Experimental results are explained in Section 6 followed by findings in Section 7. Finally, conclusions are drawn in Section 8 and Future scope for further research in section 9 .

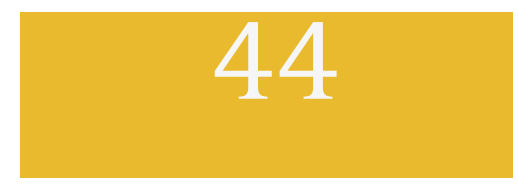


II. Need / Importance of Study: Data compression is an effective technique to lessen the amount of data needs to be communicated before transmission or stored in memory. The basic idea behind data compression is the process of eliminating redundant and irrelevant data. It represents the data in its compact form without compromising the data quality to a certain extent. Since textual data is highly available everywhere, the loss of information is not desirable and lossless compression technique is preferable. For instance, the numerals and characters in the temperature and humidity measurements, alphabets in the seismic and real time data are highly sensitive to the loss of information. The need of efficient and robust lossless compression technique motivated us to perform this study. To compress something means that you have a piece of data and you want decrease its size (Uthayakumar J. 2019).

Figure 1: Flowchart for Huffman Algorithm

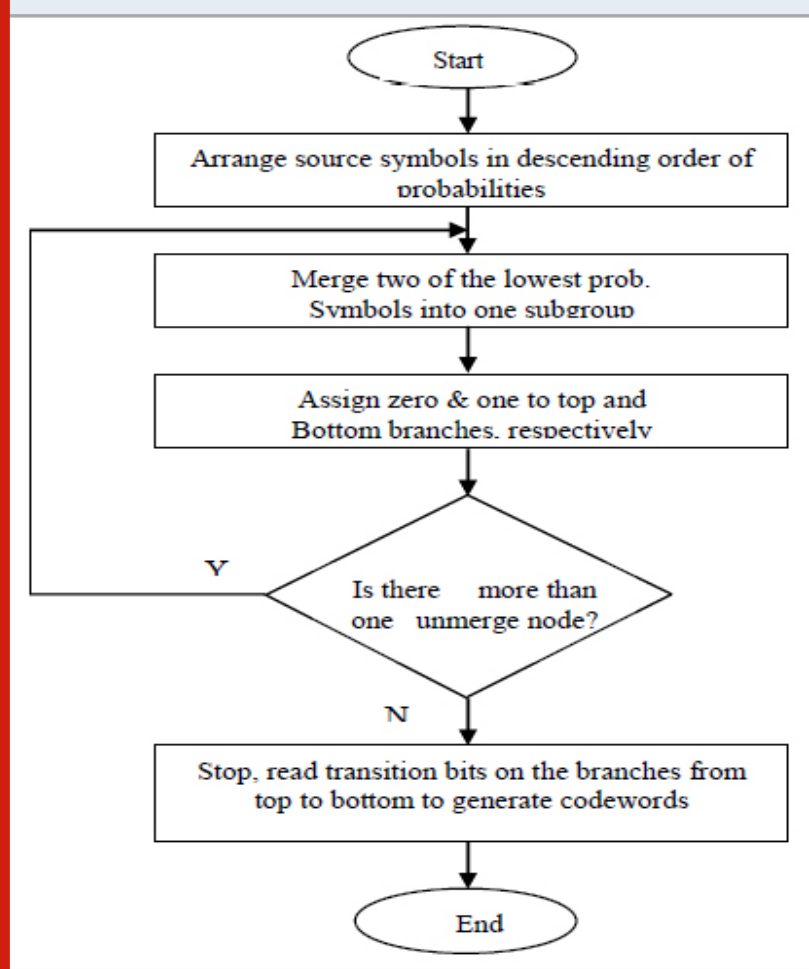

Statement of Problem: Now a days there are number of compression algorithms are available. Among them the study try to evaluate Huffman algorithm on parameters like compression ratio, compression time, and various types of file. Compression ratio: It is defined as the ratio of size of the compressed file to the size of the source file.

Compression ratio $=\mathrm{C} 2 / \mathrm{C} 1 * 100 \%$

Compression Time: It is the time taken by the algorithm to compress the file. Calculated in milliseconds (ms). (Mansouri 2020)

Hypothesis: A survey was conducted on different types of data formats and Huffman algorithms. This survey was conducted in order to check the efficiency of algorithm with respect to its corresponding data types. The data used in this work included .DOC, .TXT, .DOCX, .MDB and .XLS files. Experiments including various types of data with Huffman compression for compressing data were conducted. The purpose of these experiments was to conclude that the Huffman algorithm best suited to text data (Khairi 2018).

Table 1. Specification of the platform system used for execution of program

\begin{tabular}{|l|c|}
\hline DESKTOP & ThinkCenter Lenovo \\
\hline RAM & DD3 - 4 GB \\
\hline Processor Type & Inter core2 Due E 7500 \\
\hline No. of Cores of Processor & 2 \\
\hline Clock speed of Processor & $3 \mathrm{GHz}$ \\
\hline Cache of Processor & Windows $864-$ bit \\
\hline Operating System & \\
\hline
\end{tabular}

\section{RESEARCH METHODOLOGY}

Huffman coding is used for lossless data compression. The term entropy refers to the use of a variable length code for encoding a source symbol like character in file. The variable-length code table has been derived in a particular way, based on the estimated probability of occurrence for each possible value of the source symbol. In Huffman coding table is created incorporating the no of occurrences of an individual symbol, this table is known as frequency table. This table is arranged in particular order and then tree is generated from that table, in this tree high frequency symbols are assigned codes which have fewer bits, and less frequent symbols are assigned codes with many bits. In this way the code table is generated. We implemented Huffman algorithm in python on system with specification mentioned in table 1.

\section{RESULTS AND DISCUSSION}

The experimental results of the implemented Huffman algorithm for compression ratio and Compression time are depicted in Table2.

Findings: Huffman in 1952 proposed an sophisticated sequential algorithm which generates optimal prefix codes in $\mathrm{O}(\mathrm{nlog} n)$ time. In fact the algorithm needs only linear time provided that the frequencies of appearances are sorted in advance. There have been widespread researches on analysis, implementation issues and improvements of the Huffman coding theory in a variety of applications.

Texts are always compressed with lossless compression algorithms. This is because a loss in a text will change its original meaning. Repeated data is important in text compression. If a text contains repeated data, it can be 
compressed to a high ratio. This is due to the fact that compression algorithms generally remove repeated data. In order to evaluate the compression algorithms on the text data, a comparison between Huffman coding algorithms for different types of text files with different capacities has been performed. Experimental results showed that the compression ratio of the Huffman coding, and the performance of the Huffman coding is better.

\begin{tabular}{|c|c|c|c|c|c|}
\hline Sr. No. & Type of File & Extension & $\begin{array}{l}\text { Size of File } \\
\text { (in Bytes) }\end{array}$ & $\begin{array}{c}\text { Compression } \\
\text { Ratio }\end{array}$ & $\begin{array}{c}\text { Compression } \\
\text { Time }\end{array}$ \\
\hline 1 & Rich text document & doc & 96256 & 0.56383 & 180 \\
\hline 2 & Spreadsheet document & .xls & 51200 & 0.52 & 90 \\
\hline 3 & Rich text document & doc & 34816 & 0.382353 & 60 \\
\hline 4 & Database file &.$m d b$ & 528384 & 0.263566 & 250 \\
\hline 5 & Database file &. $\mathrm{mdb}$ & 212992 & 0.206731 & 160 \\
\hline 6 & Text document & txt & 7168 & 0.714286 & 20 \\
\hline 7 & Spreadsheet document & .xls & 102400 & 0.6 & 260 \\
\hline 8 & Rich text document & doc & 45056 & 0.454545 & 100 \\
\hline 9 & Rich text document & docx & 139264 & 0.580882 & 320 \\
\hline 10 & Text document & txt & 47104 & 0.630435 & 130 \\
\hline
\end{tabular}

\section{CONCLUSION}

Compression is an important technique in the computing field. With the help of it we can reduce the size of data and transmitting and storing the reduced data on the Internet and storage devices are faster and cheaper than uncompressed data. In this paper we have focused on Huffman algorithms in order to make clear their differences from various points of view such as implementation, compression ratio, and performance. We have explained these algorithms in detail, implemented, and tested using different file types. From implementation point of view, Huffman algorithm is easier, while Huffman algorithm needs less compression time. This means that for some applications that time is important such as real-time applications, Huffman algorithm can be used. It can also be concluded that as per the content of the original file, the performance of the algorithm varies.

Future Scope For Further Research: In order to achieve much more performance compared to software implementation, algorithms can be implemented on hardware platform such as FPGAs using parallel processing techniques. This is our future work. In future, more compression algorithms both lossy and lossless can be implemented on a larger test bed which includes audio, video and image data. And then a system can be implemented which will detect the file type and then depending on file type it will choose the appropriate compression technique for the file.

\section{REFERENCES}

Boris Ryabko, Published: 29 May 2019, “Time-Universal Data Compression”, Novosibirsk, Russia, www.mdpi. com/journal/algorithms [1-10]

Deloula Mansouri , Xiaohui Yuan * and Abdeldjalil Saidani, Published: 20 April 2020, “A New Lossless
DNA Compression Algorithm Based on A Single-Block Encoding Scheme”, Wuhan 430070, China, www.mdpi. com/journal/algorithms,[1-18]

Isamu Furuya, and Takuya Kida, Published: 8 August 2019, "Compaction of Church Numerals", Sapporo 060-0814, Japan, www.mdpi.com/journal/algorithms, [1-16]

Jérémy Barbay, Published: 31 December 2019, “Optimal Prefix Free Codes with Partial Sorting”, Santiago, Chile, www.mdpi.com/journal/algorithms, [1-21]

Liat Rozenberg, Sagi Lotan and Dan Feldman, Published: 11 December 2019, "Finding Patterns in Signals Using Lossy Text Compression”, Brisbane, Australia, www. mdpi.com/journal/algorithms, 1-18

M. Jamuna Rani, C. Vasanthanayaki, (2020), "Network condition based multi-level image compression and transmission in WSN", Salem, India, Computer Communications,317-324

Nor Alina Khairi, Asral Bahari Jambek and Nor Asilah Khairi, Oct 2018, "Design of a Huffman Data Encoder Architecture”, Perlis, Malaysia, International Journal of Nanoelectronics and Materials, [517-528]

Romain Azaïs, Published: 29 August 2019, "Nearest Embedded and Embedding Self-Nested Trees", Lyon, France, www.mdpi.com/journal/algorithm, 1-16

Sebastian Götschel and MartinWeiser, Published: 17 September 2019, "Compression Challenges in Large Scale Partial Differential Equation Solvers”, Berlin, Germany, www.mdpi.com/journal/algorithms, 1-26 Uthayakumar J, Vengattaraman T , Dhavachelvan P, (2019), “A new lossless neighborhood indexing sequence (NIS) algorithm for data compression in wireless sensor networks“, Puducherry, India , Ad Hoc Networks $149-157$ 